Tropical Journal of Pharmaceutical Research October 2020; 19 (10): 2091-2096

ISSN: $1596-5996$ (print); 1596-9827 (electronic) (C) Pharmacotherapy Group, Faculty of Pharmacy, University of Benin, Benin City, 300001 Nigeria

\title{
Krüppel-like factor 8 promotes aerobic glycolysis in prostate cancer cells by regulating AKT/mTOR signaling pathway
}

\author{
Cunming Zhang ${ }^{1}$, Song Chen ${ }^{1}$, Lide Song ${ }^{2}$, Haibo Ye ${ }^{1}$, Junwei Wang ${ }^{1 *}$ \\ ${ }^{1}$ Department of Urinary Surgery, The Affiliated Wenling Hospital of Wenzhou Medical University, Taizhou City, Zhejiang \\ Province 317500, 'Department of Urinary Surgery, Zhuji People's Hospital, Shaoxing City, Zhejiang Province 311800, China
}

*For correspondence: Email: weijun_wang123@163.com; Tel: +86-576-89668155

Sent for review: 10 August 2020

Revised accepted: 30 September 2020

\begin{abstract}
Purpose: To investigate the effects of Krüppel-like factor 8 (KLF8) in prostate cancer (PCa) cell viability and glycolysis, and explore its role as a regulatory factor.

Methods: Immunoblot assays were conducted to assess the expression of KLF8 and proteins in AKT/mTOR pathway in PCa cell lines PC-3 and DU145. Cell Counting Kit-8 assays were performed to assess the effect of KLF8 on PCa cell viability. The glycolysis capacity of PCa cells was determined by measuring the levels of glucose intake, lactic acid production, and cellular ATP levels.

Results: Depletion of KLF8 decreased the survival of PCa cells in vitro $(p<0.05)$. KLF8 depletion also inhibited aerobic glucose metabolism in PCa cells $(p<0.05)$. Further studies confirmed that KLF8 contributed to the growth and glycolysis of PCa cells via the regulation of AKT/mTOR pathway.

Conclusion: KLF8 regulates glycolysis in PCa cells by regulating AKT/mTOR signaling pathway and is thus a promising therapeutic target for $P C a$ treatment.
\end{abstract}

Keywords: Krüppel-like factor 8 (KLF8), Prostate cancer (PCa), Aerobic glucose, AKT/mTOR signaling pathway, Therapeutic target

\begin{abstract}
This is an Open Access article that uses a funding model which does not charge readers or their institutions for access and distributed under the terms of the Creative Commons Attribution License (http://creativecommons.org/licenses/by/4.0) and the Budapest Open Access Initiative (http://www.budapestopenaccessinitiative.org/read), which permit unrestricted use, distribution, and reproduction in any medium, provided the original work is properly credited.
\end{abstract}

Tropical Journal of Pharmaceutical Research is indexed by Science Citation Index (SciSearch), Scopus, International Pharmaceutical Abstract, Chemical Abstracts, Embase, Index Copernicus, EBSCO, African Index Medicus, JournalSeek, Journal Citation Reports/Science Edition, Directory of Open Access Journals (DOAJ), African Journal Online, Bioline International, Open-J-Gate and Pharmacy Abstracts

\section{INTRODUCTION}

Prostate cancer $(\mathrm{PCa})$ is a malignant tumor caused by excessive proliferation of prostatic epithelial cells [1]. Its morbidity is the second highest among male malignant tumors [2]. As age increases, the morbidity of PCa increases [3]. Prostatic adenocarcinoma originating from prostatic epithelial cells causes over $95 \%$ of all $\mathrm{PCa}$ [4]. For early PCa, androgen ablation is a successful treatment method [5]. For the treatment of advanced $\mathrm{PCa}$, chemoradiotherapy has limited efficacy and significant side effects [6]. A variety of targeted therapy drugs are used in the clinic or are in the clinical research stage [7]. However, additional effective therapeutic targets need to be identified.

The Krüppel-like factor family (KLF) are transcription factors with homology in three zincfinger DNA binding regions of $\mathrm{C} 2-\mathrm{H} 2$ and are involved in tumorigenesis in different types of 
cancer [8]. Krüppel-like factor 8 (KLF8) belongs to the KLF family [9]. KLF8 is regulated by NEDD4-mediated ubiquitination [10]. KLF8 is highly expressed in multiple types of cancers and its expression levels are correlated with the cancer's progression [11]. In hepatocellular carcinoma (HCC), KLF8 regulates VEGFA expression and angiogenesis and therefore promotes the development of HCC [12]. It also induces the EMT of pancreatic cancer cells via activating FHL2 [13].

KLF8 regulates the development and metastasis of several types of tumors [14]. However, its possible role in $\mathrm{PCa}$ is still unclear. A previous study reported that KLF8 expression is correlated with a poor prognosis in gastric cancer [15]. KLF8 affects glycolysis in cancer cells, activates GLUT4 promoter activity, and further promotes the development of gastric cancer [16]. However, whether KLF8 promotes $\mathrm{PCa}$ progression via affecting glucose metabolism in PCa cells needs further experimental evidence.

Herein, we show that KLF8 promotes the survival of $\mathrm{PCa}$ cells by regulating the activity of the Akt/mTOR pathway and increasing glycolysis. This study suggests that KLF8 could serve as a novel therapeutic target for the treatment of $\mathrm{PCa}$.

\section{EXPERIMENTAL}

\section{Inhibitors and antibodies}

The AKT/PI3K inhibitor LY294002 (CAS\#: 154447-36-6, R\&D Systems, Minneapolis, $M N$, USA) was administered at a final concentration of $20 \mu \mathrm{M}$ in PCa cells for 24 hours. The antibodies used in this study are shown in Table 1.

\section{Cell transfection}

The PC-3 and DU145 human PCa cells (ATCC, Manassas, VA, USA) were maintained in RPMI-
1640 culture medium supplemented with $10 \%$ of fetal bovine serum and incubated at $37^{\circ} \mathrm{C}$ in a $5 \% \mathrm{CO}_{2}$ incubator.

pcDNA3.1-KLF8 plasmids were constructed via RT-PCR amplification (5'-ATTAGAATTCGCC ACCATGGTCGATATGGATAAACTCATA-3' and 5'-ACCTCTCGAGTCACATGGTGTCATGGCG

$A C-3$ ') and cloned into the pcDNA3.1 vector. pLKO.1-KLF8 shRNA plasmids (sh1\#KLF8 and sh2\#KLF8) and p-LKO.1-vector shRNA plasmids were obtained from Addgene (Watertown, MA, USA). For the in vitro experiments, PC-3 and DU145 cells were transfected with $\mathrm{p}$-LKO.1-KLF8 shRNA plasmids to deplete the expression of KLF8. Scrambled shRNA plasmids were used as a control. The transfection was conducted using Lipofectamine 2000 (Invitrogen, Carlsbad, CA, USA) according to manufacturer's instructions.

\section{Immunoblotting}

Proteins were separated using SDS-PAGE and transferred onto PVDF membranes (Millipore Corporation, Bedford, MA, USA). The membranes were subsequently blocked in $5 \%$ fat-free milk in TBST buffer and incubated with specific primary antibodies against KLF8, p-AKT, AKT, p-mTOR, mTOR, and $\beta$-actin at $4^{\circ} \mathrm{C}$ overnight. After washing to remove non-specific binding, the membrane was treated with HRPlabeled secondary antibody in TBST buffer. Then, the blots were visualized using an ECL kit. The relative protein levels were quantified using ImageJ (NIH, Bethesda, MD, USA).

\section{Cell viability assay}

PC-3 and DU145 cells were incubated in 96-well plates with $100 \mu \mathrm{L}$ culture medium per well. Cell viability was detected using a Cell Counting Kit-8 (CCK-8). CCK-8 solution was added to the cells and incubated at $37^{\circ} \mathrm{C}$ for 3 hours. Then, absorbance was measured using a Bio-Rad microplate reader (Richmond, CA, USA).

Table 1: Antibodies

\begin{tabular}{ll}
\hline Antibody & Information \\
\hline Anti-KLF8 antibody & $1: 1000$ dilution, ab168527, Abcam, \\
& Cambridge, UK \\
Anti-AKT antibody & $1: 1000$ dilution, ab8805, Abcam, \\
& Cambridge, UK \\
Anti- $\beta$-actin antibody & $1: 1000$ dilution, ab8226, Abcam, \\
& Cambridge, UK \\
Anti-p-AKT (phospho T308) antibody & $1: 1000$ dilution, ab38449, Abcam, \\
& Cambridge, UK \\
Anti-mTOR antibody & $1: 500$ dilution, ab32028, Abcam, \\
Anti-p-mTOR (phospho S2448) antibody & Cambridge, UK \\
& $1: 1000$ dilution, ab84400, Abcam, \\
& Cambridge, UK \\
\hline
\end{tabular}


Cell viability was displayed as a percentage of the OD value in the experimental group compared to the control group at $490 \mathrm{~nm}$.

\section{Cell glycolysis test}

The glycolysis levels of PCa cells were evaluated according to the glucose intake (ab136955), lactic acid production (ab83429), and cellular ATP levels (ab83355), which were measured using the corresponding kits from Abcam Company (Cambridge, UK). All experiments were conducted according to the respective instructions.

\section{Statistical analysis}

Statistical analysis was performed using GraphPad (San Diego, CA, USA). Data were representative of at least three independent experiments and presented as mean \pm SD. Statistical analysis was conducted using the Student's t-test and $p<0.05$ was considered statistically significant.

\section{RESULTS}

\section{KLF8 depletion decreased the viability of PCa cells}

KLF8 expression decreased after the transfection of its shRNA plasmids in both PC-3 and DU145 cells, confirming effective depletion (Figure 1A).
A
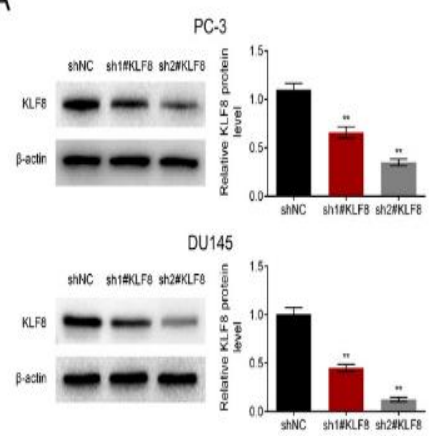

B

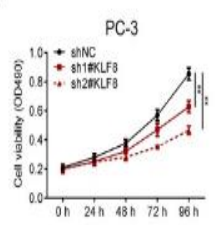

DU145

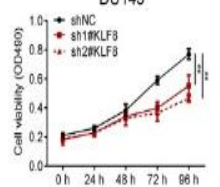

Figure 1: KLF8 depletion decreased the viability of $\mathrm{PCa}$ cells in vitro. (A) Immunoblot assays showed the expression of KLF8 and $\beta$-actin after the transfection of the indicated plasmids in $\mathrm{PC}-3$ and DU145 cells. (B). CCK-8 assays showed the difference of cell viability in PC-3 and DU145 cells after the transfection of the indicated plasmids. Results are presented as mean \pm SD; ${ }^{* *} p<0.01$

Subsequently, the effects of KLF8 depletion on the viability of $\mathrm{PCa}$ cells was detected using
CCK-8 assays. Interestingly, KLF8 depletion significantly inhibited the viability of $\mathrm{PC}-3$ and DU145 cells in vitro (Figure 1B). Therefore, these data revealed the decreased cell viability induced by KLF8 depletion.

\section{Depletion of KLF8 impaired glycolysis in $\mathrm{PCa}$ cells in vitro}

Interestingly, glucose intake levels dramatically decreased after KLF8 depletion in PC-3 and DU145 cells (Figure 2 A). Moreover, lactate production levels and cellular ATP levels also decreased after the transfection of KLF8 shRNA plasmids in PC-3 and DU145 cells (Figure 2 B and C). Therefore, KLF8 depletion impaired glycolysis in PCa cells in vitro.
A

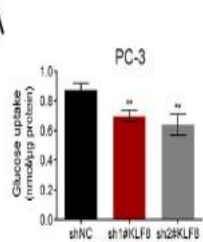

DU145

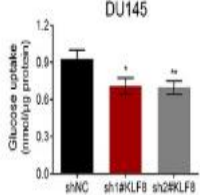

B

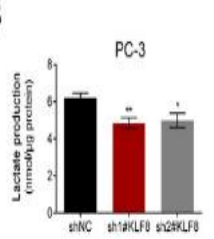

DU145

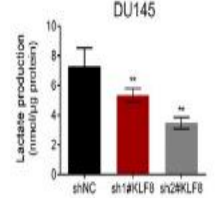

C

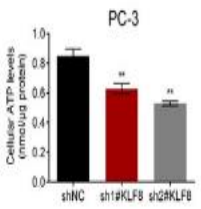

DU145

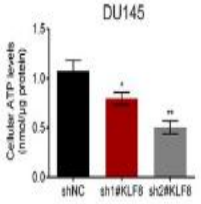

Figure 2: KLF8 depletion impaired glycolysis in PCa cells in vitro. (A - C) The levels of glucose uptake (A), lactate production (B), and cellular ATP levels (C) were detected in PC-3 and DU145 cells after transfection of the indicated plasmids. Results are presented as mean $\pm \mathrm{SD} ;{ }^{*} p<0.05,{ }^{* *} p<0.01$

\section{KLF8 regulated $A K T / m T O R$ pathway in $\mathrm{PCa}$ cells}

The AKT/mTOR pathway is critical in the metabolic regulation of cancer cells. The expression and phosphorylation levels of AKT and mTOR were detected through immunoblot assays. Interestingly, the phosphorylation of AKT was inhibited in both PC-3 and DU145 cells after KLF8 depletion (Figure $3 \mathrm{~A}$ and $\mathrm{B}$ ). Meanwhile, mTOR phosphorylation levels were also decreased in KLF8-depleted PC-3 and DU145 cells (Figures $3 A$ and $B$ ). However, the total expression levels of AKT and mTOR in control or KLF8 depleted PCa cells were unchanged (Figures $3 \mathrm{~A}$ and $\mathrm{B}$ ). Collectively, KLF8 regulated the AKT/mTOR pathway in PCa cells.

Trop J Pharm Res, October 2020; 19(10): 2093 
KLF8 contributes to the growth and glycolysis of $\mathrm{PCa}$ cells by regulating AKT/mTOR pathway

Immunoblot assays showed that KLF8 overexpression increased the phosphorylation levels of AKT and mTOR in PC-3 cells, consistent with the previous results (Figure 4A). Importantly, LY294002 treatment blocked the increase of the phosphorylation levels of AKT and mTOR caused by KLF8 overexpression in PC-3 cells (Figure $4 \mathrm{~A}$ ). However, the treatment of LY294002 had no obvious effects on KLF8 expression levels (Figure 4A). Therefore, LY294002 treatment effectively inhibited the AKT/mTOR pathway.
A

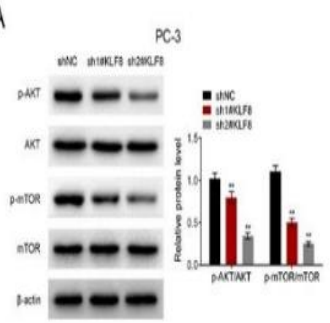

B

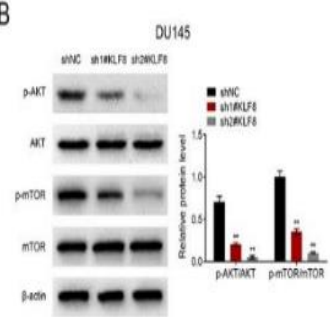

Figure 3: KLF8 regulated the AKT/mTOR pathway in $\mathrm{PCa}$ cells. (A, B) Immunoblot assays revealed the expression level of the indicated proteins after transfection of sh1\#KLF8, sh2\#KLF8, and sh-vector plasmids in PC-3 (A) and DU145 (B) cells. Results are presented as mean $\pm \mathrm{SD},{ }^{* *} p<0.01$

Subsequently, the effects of KLF8 overexpression and LY294002 treatment on PC3 cell growth were assessed. Through CCK-8 assays, KLF8 overexpression dramatically promoted the growth of PC-3 cells, whereas LY294002 treatment significantly suppressed the growth of KLF8 overexpressing PC-3 cells (Figure $4 \mathrm{~B}$ ).

The effects of KLF8 and LY294002 on PC-3 cell glycolysis were measured. Similarly, the levels of glucose intake, lactic acid production, and cellular ATP were upregulated after KLF8 overexpression (Figure $4 \mathrm{C}$ ). However, the LY294002 treatment reversed the increased glucose intake, lactic acid production, and cellular ATP caused by KLF8 overexpression in PC-3 cells (Figure $4 \mathrm{C}$ ). Therefore, KLF8 regulated the growth and glycolysis of $\mathrm{PCa}$ cells through the AKT/mTOR pathway.

\section{DISCUSSION}

In recent years, the morbidity of $\mathrm{PCa}$ has increased [17]. In 2018, approximately 1,300,000 $\mathrm{PCa}$ cases were newly reported worldwide, accounting for $13.5 \%$ of the morbidity of malignant tumors in males, and approximately 360,000 deaths occurred, accounting for $6.7 \%$ of cancer mortality for male patients [18]. Patients are often in an advanced stage of $\mathrm{PCa}$ when they are diagnosed due to the lack of significant early symptoms [6].

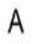

A

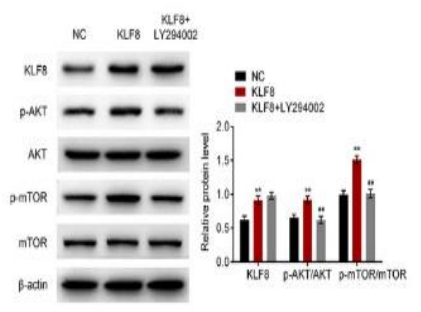

C

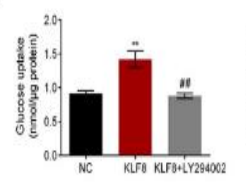

B
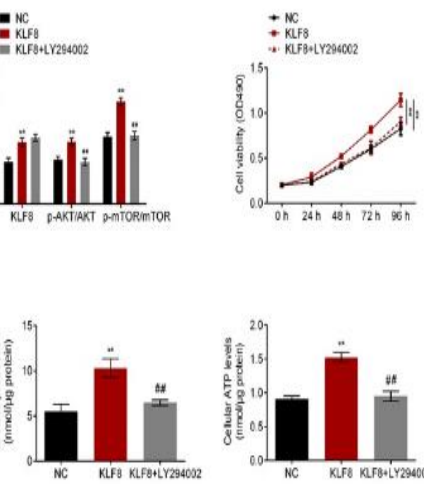

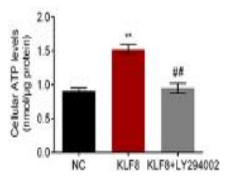

Figure 4: KLF8 contributes to the growth and glycolysis of $\mathrm{PCa}$ cells via regulating the AKT/mTOR pathway. (A). Immunoblot assays revealed the expression level of the indicated proteins after LY294002 treatment in control or KLF8 overexpression PC-3 cells. (B). CCK-8 assays showed the cell viability of PC-3 cells after treatment with the indicated plasmids or drugs. (C). Glucose uptake, lactate production, and cellular ATP levels were detected in PC-3 cells after treatment with the indicated plasmids or drugs. Results are presented as mean $\pm S D ;{ }^{* *} p<0.01, \# \# p 0.001$

By this time, tumor cells spread outside the prostate, and the prognosis is often poor. Finding novel and effective therapeutic targets is urgently needed to combat PCa [19]. In this study, the role of a KLF family transcription factor, KLF8, was examined in the regulation of $\mathrm{PCa}$ cell growth and glycolysis. The data confirmed the involvement of KLF8 in the development of $\mathrm{PCa}$ and suggested KLF8 could serve as a promising $\mathrm{PCa}$ molecular target.

The proteins in the KLF family widely affect the development and metastasis of multiple cancers [20]. KLF8 also regulates the development of cancers and correlates with the prognosis and clinical features of multiple cancers [21]. KLF8 contributes to LUAD metastasis via promoting the EMT process [20]. KLF8 promotes the cancer stem cell-like phenotypes in osteosarcoma via the miR-429-SOX2 axis [22]. KLF8 overexpression also contributes to the growth of lung cancer cells via stimulating JMJD2A expression [21]. Here, the critical role of KLF8 in $\mathrm{PCa}$ cells was revealed. These studies, together with this study, suggest KLF8 could serve as an important cancer molecular target. 
The regulation of KLF8 on the AKT/mTOR pathway in $\mathrm{PCa}$ cells was also revealed. The AKT/mTOR pathway widely affects the progression and metastasis of tumors [23]. Previous studies confirmed that in $\mathrm{PCa}$, microRNA-381 facilitates autophagy and apoptosis via the RELN-mediated AKT/mTOR pathway [23]. EHMT2 promotes the progression of $\mathrm{PCa}$ also through the AKT/mTOR pathway [24]. KLF8, as a transcription factor, could promote the growth of $\mathrm{PCa}$ cells via this pathway. Thus, it is important to find the proteins transcriptionally regulated by KLF8 in this pathway.

Aerobic glycolysis is a key feature of tumor cells. Several signaling pathways are involved in the regulation of glycolysis and other metabolic activities, such as the Akt/MAPK and Akt/mTOR pathways [16]. A variety of oncogenes regulate cancer glycolysis through these signaling pathways, thereby promoting tumor growth [25]. KLF8 could also regulate Akt/mTOR to promote aerobic glycolysis and cell growth of PCa. Therefore, KLF8 targeted therapy may inhibit aerobic glycolysis in PCa and further inhibit the growth and metastasis of tumor cells.

\section{CONCLUSION}

KLF8 regulates the viability and glycolysis of $\mathrm{PCa}$ cells. It also regulates the AKT/mTOR pathway in $\mathrm{PCa}$ cells, thus confirming that KLF8 promotes the growth and glycolysis of $\mathrm{PCa}$ cells via the AKT/mTOR pathway. Therefore, KLF8 is a promising therapeutic target for $\mathrm{PCa}$ treatment.

\section{DECLARATIONS}

\section{Conflict of interest}

No conflict of interest is associated with this work.

\section{Contribution of authors}

We declare that this work was done by the authors named in this article and all liabilities pertaining to claims relating to the content of this article will be borne by the authors. Cunming Zhang and Song Chen designed the study, supervised the data collection, and analyzed the data. Lide Song interpreted the data and prepared the manuscript for publication. Haibo Ye and Junwei Wang supervised the data collection, analyzed the data, and reviewed the draft of the manuscript. All authors have read and approved the manuscript.

\section{Open Access}

This is an Open Access article that uses a funding model which does not charge readers or their institutions for access and distributed under the terms of the Creative Commons Attribution License (http://creativecommons.org/licenses/by/ 4.0) and the Budapest Open Access Initiative (http://www.budapestopenaccessinitiative.org/rea d), which permit unrestricted use, distribution, and reproduction in any medium, provided the original work is properly credited.

\section{REFERENCES}

1. lacovelli $R$, Ciccarese C, Caffo O, De Giorgi U, Tucci M, Mosillo C, Bimbatti D, Pierantoni F, Maines F, Casadei $C$ et al. The prognostic value of pain in castrationsensitive prostate cancer. Prostate Cancer Prostatic Dis 2020. https://doi.org/10.1038/s41391-020-0255-x

2. Kido LA, Hahm ER, Kim SH, Baseggio AM, Cagnon VHA, Singh SV, Marostica MR, Jr. Prevention of Prostate Cancer in Transgenic Adenocarcinoma of the Mouse Prostate Mice by Yellow Passion Fruit Extract and Antiproliferative Effects of Its Bioactive Compound Piceatannol. J Cancer Prev 2020; 25(2): 87-99.

3. Chung MS, Cho NH, Kim J, Jo Y, Yoon BI, Lee SH. Predicting Insignificant Prostate Cancer: Analysis of the Pathological Outcomes of Candidates for Active Surveillance according to the Pre-International Society of Urological Pathology (Pre-ISUP) 2014 Era Versus the Post-ISUP2014 Era. World J Mens Health 2020.

4. Ding Y, Li N, Dong B, Guo W, Wei $H$, Chen Q, Yuan $H$, Han $Y$, Chang $H$, Kan $S$ et al. Chromatin remodeling ATPase BRG1 and PTEN are synthetic lethal in prostate cancer. J Clin Invest 2019; 129(2): 759-773.

5. Pereira-Nunes A, Simoes-Sousa S, Pinheiro C, MirandaGoncalves V, Granja S, Baltazar F. Targeting lactate production and efflux in prostate cancer. Biochim Biophys Acta Mol Basis Dis 2020: 165894.

6. Hassani S, Salek MA, Rezaei AM, Rahmani SR, Sarihi P, Ganjali MR, Norouzi $P$, Abdollahi M. A Sensitive Aptamer-Based Biosensor for Electrochemical Quantification of PSA as a Specific Diagnostic Marker of Prostate Cancer. J Pharm Sci 2020; 23: 243-258.

7. Kokal M, Mirzakhani K, Pungsrinont T, Baniahmad A. Mechanisms of Androgen Receptor Agonist- and Antagonist-Mediated Cellular Senescence in Prostate Cancer. Cancers (Basel) 2020; 12(7).

8. Bureau C, Hanoun N, Torrisani J, Vinel JP, Buscail L, Cordelier P. Expression and Function of Kruppel LikeFactors (KLF) in Carcinogenesis. Curr Genomics 2009; 10(5): 353-360.

9. Hu JH, Navas $P$, Cao $H$, Stamatoyannopoulos $G$, Song $C Z$. Systematic RNAi studies on the role of Sp/KLF factors in globin gene expression and erythroid differentiation. J Mol Biol 2007; 366(4): 1064-1073.

Trop J Pharm Res, October 2020; 19(10): 2095 
10. Lossi AM, Laugier-Anfossi $F$, Depetris D, Gecz J, Gedeon A, Kooy F, Schwartz C, Mattei MG, Croquette $M F$, Villard L. Abnormal expression of the KLF8 (ZNF741) gene in a female patient with an X;autosome translocation $t(X ; 21)(p 11.2 ; q 22.3)$ and non-syndromic mental retardation. J Med Genet 2002; 39(2): 113-117.

11. Wang $X$, Zhao J. KLF8 transcription factor participates in oncogenic transformation. Oncogene 2007; 26(3): 456461.

12. Bin Z, Ke-Yi L, Wei-Feng Z, Li-Cheng J, Xian-Bin L, Chun-Peng $X$, Dao-Ying $Y$, Shu-Wei L. Downregulation of KLF8 expression by ShRNA induces inhibition of cell proliferation in CAL27 human oral cancer cells. Med Oral Patol Oral Cir Bucal 2013; 18(4): e591-596.

13. Yi $X, L i Y$, Zai H, Long $X, L i W$. KLF8 knockdown triggered growth inhibition and induced cell phase arrest in human pancreatic cancer cells. Gene 2016; 585(1): 22-27.

14. Liang K, Liu T, Chu N, Kang J, Zhang R, Yu Y, Li D, Lu $D$. KLF8 is required for bladder cancer cell proliferation and migration. Biotechnol Appl Biochem 2015; 62(5): 628-633.

15. Zhang H, Liu L, Wang Y, Zhao G, Xie R, Liu C, Xiao X, Wu K, Nie $Y$, Zhang $H$ et al. KLF8 involves in TGF-betainduced EMT and promotes invasion and migration in gastric cancer cells. J Cancer Res Clin Oncol 2013; 139(6): 1033-1042.

16. Mao A, Zhou X, Liu Y, Ding J, Miao A, Pan G. KLF8 is associated with poor prognosis and regulates glycolysis by targeting GLUT4 in gastric cancer. J Cell Mol Med 2019; 23(8): 5087-5097.

17. Flynn L, Barr MP, Baird AM, Smyth $P$, Casey OM, Blackshields G, Greene J, Pennington SR, Hams E, Fallon PG et al. Prostate cancer-derived holoclones: a novel and effective model for evaluating cancer stemness. Sci Rep 2020; 10(1): 11329.
18. Kim M, Byun SS, Hong SK. Testosterone Replacement Therapy in Men with Untreated or Treated Prostate Cancer: Do We Have Enough Evidences? World J Mens Health 2020.

19. Abudurexiti $M$, Zhu W, Wang $Y$, Wang J, Xu W, Huang $Y$, Zhu $Y$, Shi G, Zhang $H$, Zhu $Y$ et al. Targeting CPT1B as a potential therapeutic strategy in castration-resistant and enzalutamide-resistant prostate cancer. Prostate 2020; 80(12): 950-961.

20. Jing $P$, Xie N, Zhao N, Zhu X, Li P, Gao G, Dang H, Gu Z. miR-24-3p/KLF8 Signaling Axis Contributes to LUAD Metastasis by Regulating EMT. J Immunol Res 2020; 2020(4036047. doi: 10.1155/2020/4036047.

21. Ma D, Liu H, Qin $Y$, Tian Z, Li S, Liang N. KLF8 overexpression promotes the growth of human lung cancer cells by promoting the expression of JMJD2A. Cancer Cell Int 2019; 19(258. doi: 10.1186/s12935-0190970-3.

22. Zhang L, Yang $P$, Liu Q, Wang J, Yan F, Duan L, Lin F. KLF8 promotes cancer stem cell-like phenotypes in osteosarcoma through miR-429-SOX2 signaling. Neoplasma 2020; 67(3): 519-527. doi: 10.4149/neo_2020_190711N624.

23. Liao W, Zhang Y. MicroRNA-381 facilitates autophagy and apoptosis in prostate cancer cells via inhibiting the RELN-mediated PI3K/AKT/mTOR signaling pathway. Life Sci 2020; 254(117672. doi: 10.1016/j.lfs.2020.117672.

24. Fan HT, Shi YY, Lin Y, Yang XP. EHMT2 promotes the development of prostate cancer by inhibiting PI3K/AKT/mTOR pathway. Eur Rev Med Pharmacol Sci 2019; 23(18): 7808-7815. doi: 10.26355/eurrev_201909_18990.

25. Feng $X$, Wang CX, Ou ZY. TGF-beta 1 and IL-10 expression in epithelial ovarian cancer cell line A2780. Trop J Pharm Res 2015; 14(12): 2179-2185. doi: 10.4314/tjpr.v14i12.4. 